\title{
On Faster Convergence of the Bisection Method for all Triangles
}

\author{
By Martin Stynes*
}

\begin{abstract}
Let $\triangle A B C$ be a triangle with vertices $A, B$, and $C$. It is "bisected" as follows: choose a/the longest side (say $A B$ ) of $\triangle A B C$, let $D$ be the midpoint of $A B$, then replace $\triangle A B C$ by two triangles $\triangle A D C$ and $\triangle D B C$.

Let $\Delta_{01}$ be a given triangle. Bisect $\Delta_{01}$ into two triangles $\Delta_{11}$ and $\Delta_{12}$. Next bisect each $\Delta_{1 i}, i=1,2$, forming four new triangles $\Delta_{2 i}, i=1,2,3,4$. Continue thus, forming an infinite sequence $T_{j}, j=0,1,2, \ldots$, of sets of triangles, where $T_{j}=\left\{\Delta_{j i}: 1\right.$ $\left.\leqslant i \leqslant 2^{j}\right\}$. Let $m_{j}$ denote the mesh of $T_{j}$. It is shown that there exists $N=N\left(\Delta_{01}\right)$ such that, for $j \geqslant N, m_{2 j} \leqslant(\sqrt{3} / 2)^{N}(1 / 2)^{j}-N_{m_{0}}$, thus greatly improving the previous best known bound of $m_{2 j} \leqslant(\sqrt{3} / 2) j_{m_{0}}$.

It is also shown that only a finite number of distinct shapes occur among the triangles produced, and that, as the method proceeds, $\Delta_{01}$ tends to become covered by triangles which are approximately equilateral in a certain sense.
\end{abstract}

1. Introduction and Summary. Let $\triangle A B C$ be a triangle with vertices $A, B$, and $C$. We define the procedure for "bisecting" $\triangle A B C$ as follows: choose a/the longest side (say $A B$ ) of $\triangle A B C$, let $D$ be the midpoint of $A B$, then divide $\triangle A B C$ into the two triangles $\triangle A D C$ and $\triangle D B C$.

Let $\Delta_{01}$ be a given triangle. Bisect $\Delta_{01}$ into two triangles $\Delta_{11}$ and $\Delta_{12}$. Next bisect each $\Delta_{1 i}, i=1,2$, forming four new triangles $\Delta_{2 i}, i=1,2,3,4$. Continue thus, forming an infinite sequence $T_{j}, j=0,1,2, \ldots$, of sets of triangles, where $T_{j}$ $=\left\{\Delta_{j i}: 1 \leqslant i \leqslant 2^{j}\right\}$. Define $m_{j}$, the mesh of $T_{j}$, to be the length of the longest side among the sides of the triangles of $T_{j}$. Clearly $0<m_{j+1} \leqslant m_{j}$, for all $j \geqslant 0$. It is known [2], [1] that $m_{j} \rightarrow 0$ as $j \rightarrow \infty$.

In [1, Theorem 3.1] an explicit bound is obtained for the rate of convergence of $m_{j}: m_{j} \leqslant(\sqrt{3} / 2)^{[j / 2]} m_{0}, j \geqslant 0$, where $[x]$ denotes the integer part of $x$. This may be written as $m_{2 j} \leqslant(\sqrt{3} / 2)^{j} m_{0}, j \geqslant 0$. In [1] it is also mentioned that computer experiments indicate that in many cases this bound is unrealistically high. This prompted the results of [3] where it was shown that if $\Delta_{01}$ lay in any one of four sets of similarity classes of triangles, then $m_{2 j} \leqslant(\sqrt{3} / 2)(1 / 2)^{j-1} m_{0}$, for $j \geqslant 1$. Note however that in [3] the inequality was not written in this form; see [3, Corollaries 1 and 2]. In the present paper we show that for any $\Delta_{01}$ there exists a positive integer $N$ depending only on $\Delta_{01}$ such that

Received April 10, 1979; revised February 28, 1980.

AMS (MOS) subject classifications (1970). Primary 50B30, 50B15; Secondary 41 A63, $65 \mathrm{~N} 30,55 \mathrm{C} 25$.

*This paper was written while the author was at University College, Cork, Ireland. 


$$
m_{2 j} \leqslant(\sqrt{3} / 2)^{\min \{j, N\}}(1 / 2)^{\max \{j-N, 0\}_{m_{0}}}, \text { for } j \geqslant 0 .
$$

For $j \geqslant N$ this becomes $m_{2 j} \leqslant(\sqrt{3} / 2)^{N}(1 / 2)^{j-N} m_{0}$, thus generalizing [3, Corollaries 1 and 2]. It is also a great improvement on the result of [1] for $j$ large. We have the interesting result of Corollary 1 below that for any $\Delta_{01}$ the bisection method yields only a finite number of distinct shapes among all the triangles in the $T_{j}, j=0$, $1,2, \ldots$ Finally Corollary 2 shows that the shapes produced tend to be closer to equilateral than the original $\Delta_{01}$.

2. Results. All notation used is consistent with that of [3]. The results of this paper can also be proven using the methods of [2], [3] (i.e., by consideration of angles in triangles) but the technique below seems simpler.

Given two triangles $\Delta$ and $\Delta^{\prime}$, we write $\Delta \sim \Delta^{\prime}$ to indicate that the triangles are similar.

Definitions. Given a triangle $\Delta$, define $d(\Delta)$, the diameter of $\Delta$, to be length of the longest side of $\Delta$. Define $t(\Delta)$ to be (area $\Delta) / d^{2}(\Delta)$. Note that if $\Delta \sim \Delta^{\prime}$, then $t(\Delta)=t\left(\Delta^{\prime}\right)$. (This similarity invariant $t$ is closely related to J. H. C. Whitehead's "relative thickness of simplexes" [5, pp. 811, 812].)

Note that when a triangle is bisected, its area is bisected. This fact is used implicitly many times in what follows.

Definition. We say that $\Delta_{01}$ is good if it has an associated positive integer $N$ such that

$$
m_{2 j} \leqslant(\sqrt{3} / 2)^{\min \{j, N\}}(1 / 2)^{\max \{j-N, 0\}} m_{0}, \quad \text { for } j \geqslant 0 .
$$

For the rest of this paper we always assume that we are working with the smallest such $N$ associated with a given $\Delta_{01}$.

Remarks. Clearly all triangles generated from a good $\Delta_{01}$, by the bisection method, are also good, using the result of [1] that $m_{2 j} \leqslant(\sqrt{3} / 2)^{j} m_{0}, j \geqslant 0$, for any $\Delta_{01}$. In [3, Corollary 1 (i)] it was shown that for $\Delta_{01}=\triangle A B C$, as in Figure 1 satisfying (length of $A C \Rightarrow$ ) $A C \leqslant B C \leqslant A B, A C \geqslant \max \{C D, A D\}$, and $C D \geqslant C F$, where $D, F$ are the midpoints of $A B, B C$ respectively, we have $m_{2 j} \leqslant$ $(\sqrt{3} / 2)^{\min \{j, 1\}}(1 / 2)^{\max \{j-1,0\}} m_{0}$, for $j \geqslant 0$, so such triangles are good. We note that these inequalities were expressed differently in [3]. In [3] three other classes of triangles satisfying conditions on the length of their sides and medians were considered and similar inequalities for $m_{2 j}$ were proven; however, these three classes can be shown to be subclasses of the one specified above (this passed unnoticed in [3]).

LEMMA 1. If $t\left(\Delta_{01}\right) \geqslant \sqrt{7} / 8$, then $\Delta_{01}$ is good.

Proof. We use the result just quoted from [3]. Take $\Delta_{01}=\triangle A B C$ in Figure 1. Assuming $A C \leqslant B C \leqslant A B$, we show that $A C \geqslant \max \{C D, A D\}$ and $C D \geqslant C F$.

Since $t(\Delta)$ is invariant under similarity, we may take $A B=1$. In Figure $1, C L$ is perpendicular to $A B$. Let $C L=h, A L=x$, and $L B=1-x$. Now $7 / 64 \leqslant$ $t^{2}(\triangle A B C)=h^{2} / 4$ by definition of $t(\Delta)$, so $h^{2} \geqslant 7 / 16$. Hence $1=A B^{2} \geqslant B C^{2}=$ $h^{2}+(1-x)^{2} \geqslant 7 / 16+(1-x)^{2}$, which gives $x \geqslant 1 / 4$. 


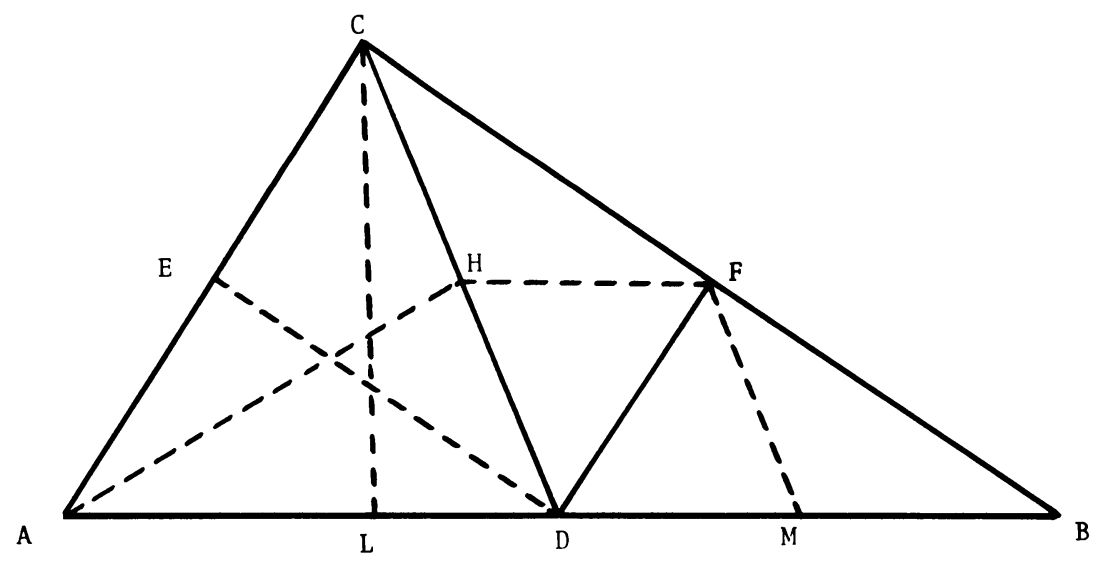

Figure 1

Now

$$
\begin{aligned}
\max \left\{C D^{2}, A D^{2}\right\} & =\max \left\{h^{2}+(1 / 2-x)^{2}, 1 / 4\right\} \\
& \leqslant h^{2}+x^{2} \quad \text { since } h^{2} \geqslant 7 / 16 \text { and } 1 / 2 \geqslant x \geqslant 1 / 4=A C^{2} .
\end{aligned}
$$

Also

$$
\begin{aligned}
C F^{2} & =B C^{2} / 4=\left(h^{2}+(1-x)^{2}\right) / 4 \\
& \leqslant h^{2} \quad \text { since } x \geqslant 1 / 4 \text { and } h^{2} \geqslant 7 / 16 \leqslant C D^{2} .
\end{aligned}
$$

By the remarks above, $\Delta_{01}=\triangle A B C$ is good.

Definitions. Using the notation of the introduction, an iteration of the bisection method is defined to be the progression from $T_{j}$ to $T_{j+1}$, for any $j \geqslant 0$. A cycle of the bisection method is defined to be two successive iterations, i.e., the progression from $T_{j}$ to $T_{j+2}$, for any $j \geqslant 0$.

LeMma 2. After one cycle of the bisection procedure, applied to $\Delta_{01}$, we have four triangles $\Delta_{2 i}, i=1,2,3,4$. Suppose that for each $i$ either $\Delta_{2 i}$ is good or $\Delta_{2 i} \sim \Delta_{01}$ with $d\left(\Delta_{2 i}\right)=d\left(\Delta_{01}\right) / 2$. Then $\Delta_{01}$ is good.

Proof. Set $N=1+\max \left\{N_{i}: \Delta_{2 i}\right.$ good with associated positive integer $\left.N_{i}\right\}$.

We show that

$$
m_{2 j} \leqslant(\sqrt{3} / 2)^{\min \{j, N\}}(1 / 2)^{\max \{j-N, 0\}} m_{0}, \quad \text { for } j \geqslant 0 .
$$

To do this we use induction on $j$.

For $j \leqslant N$, this is the $n=2$ case of [1, Theorem 3.1]. Fix $k \geqslant N$ and assume that the result holds for $j=k$. We now prove it for $j=k+1$.

Each triangle $\Delta_{2(k+1), i^{\prime}}, i^{\prime}=1,2, \ldots, 2^{2(k+1)}$, is obtained by applying $k$ bisection cycles to one of the $\Delta_{2 i}, i=1,2,3,4$. We consider the possibilities for $\Delta_{2 i}$ separately. If $\Delta_{2 i}$ is good with associated positive integer $N_{i}$, then, after $k$ bisection cycles applied to it, any resulting triangle $\Delta_{2(k+1), i^{\prime}}$ satisfies 


$$
\begin{aligned}
& d\left(\Delta_{2(k+1), i^{\prime}}\right) \leqslant(\sqrt{3} / 2)^{N_{i}}(1 / 2)^{k-N_{i}} d\left(\Delta_{2 i}\right) \\
& \leqslant(\sqrt{3} / 2)^{N_{i}+1}(1 / 2)^{k-N_{i}} m_{0} \text { by [1, Theorem 3.1] } \\
& \leqslant(\sqrt{3} / 2)^{N}(1 / 2)^{k+1-N} m_{0} \quad \text { since } N_{i}+1 \leqslant N \text {. }
\end{aligned}
$$

If $\Delta_{2 i} \sim \Delta_{01}$ with $d\left(\Delta_{2 i}\right)=d\left(\Delta_{01}\right) / 2$, then, after $k$ bisection cycles applied to it, any resulting triangle $\Delta_{2}(k+1), i^{\prime}$ satisfies

$$
d\left(\Delta_{2(k+1), i^{\prime}}\right) \leqslant(\sqrt{3} / 2)^{N}(1 / 2)^{k-N} d\left(\Delta_{2 i}\right)
$$

by the inductive hypothesis applied to $\Delta_{2 i}$ since $\Delta_{2 i} \sim \Delta_{01}$. Now use $d\left(\Delta_{2 i}\right)=$ $m_{0} / 2$ to complete the proof for $j=k+1$.

THEOREM 1. Any triangle $\Delta_{01}$ is good.

Proof. Let $S$ be the set of all triangles $\Delta_{01}$ which are not good. Assuming $S$ is not empty, set $\hat{t}=\sup \{t(\Delta): \Delta \in S\}$. By Lemma $1, \hat{t} \leqslant \sqrt{7} / 8$. Now choose $\Delta_{01} \in$ $S$ such that $t^{*} \equiv t\left(\Delta_{01}\right) \geqslant \max \left\{3 \hat{t} / 4, \hat{t}\left(1-9 \tilde{t}^{2} / 2\right)\right\}$. We show that in fact $\Delta_{01}$ is good, contradicting the assumption that $S$ is nonempty.

Take $\Delta_{01}=\triangle A B C$ in Figure 1 , with $A C \leqslant B C \leqslant A B$. Here $D, E, F, H$, and $M$ are the midpoints of $A B, A C, B C, C D$, and $B D$ respectively. When the bisection method is applied to $\triangle A B C, A B$ and $B C$ must be bisected as shown. Any of the three sides in $\triangle C A D$ may be bisected next, while in $\triangle C D F$ either $C D$ or $C F$ will be bisected since $D F=A C / 2 \leqslant B C / 2=C F$ (if $D F=C F$, we can assume that $C F$ is bisected since either choice has the same effect). This gives six possible combinations of bisections of sides. We analyze these cases separately.

Case (i): $A C$ in $\triangle C A D, C D$ in $\triangle C D F$. In this case we are in the situation of [3, Corollary 1(i)]; by the Remarks above $\triangle A B C$ is good.

Case (ii): $C D$ in $\triangle C A D, C F$ in $\triangle C D F$. This is impossible unless $C D=C F$, since $C D \geqslant A D=A B / 2 \geqslant B C / 2=C F$ and if $C D>C F$, then $C F$ cannot be bisected in $\triangle C D F$. If $C D=C F$, we proceed to Case (vi) below.

For the remaining cases we shall use the well-known formula for the length of the median $C D$ :

$$
C D^{2}=A C^{2} / 2+B C^{2} / 2-A B^{2} / 4 \text {. }
$$

Case (iii): $A C$ in $\triangle C A D, C F$ in $\triangle C D F$. Now $B C^{2} / 4=C F^{2} \geqslant C D^{2} \geqslant A D^{2} / 2$ $+B C^{2} / 2-A B^{2} / 4$, hence $A B^{2} / 2 \geqslant B C^{2}$. Thus $t(\triangle C D F)=($ area $\triangle C D F) / C F^{2}=$ (area $\triangle A B C) / B C^{2} \geqslant 2 t^{*}$. By choice of $t^{*} \triangle C D F$ is good.

When $\triangle C A D$ is bisected, we get $\triangle A D E \sim \triangle A B C$ with $d(\triangle A D E)=d(A B C) / 2$ and $\triangle C E D \sim \triangle C D F$, so $\triangle C E D$ is good. Also $\triangle D B F \sim \triangle A B C$ with $d(\triangle D B F)=$ $d(\triangle A B C) / 2$. By Lemma $2 \triangle A B C$ is good.

Case (iv): $A D$ in $\triangle C A D, C F$ in $\triangle C D F$. Here $t(\triangle C A D)=($ area $\triangle C A D) / A D^{2}=$ 
2(area $\triangle A B C) / A B^{2}=2 t^{*}$, so $\triangle C A D$ is good, and consequently the two triangles obtained from it by bisection are good.

Now $t^{*}=(A B \cdot A C \cdot \sin C \hat{A B}) /\left(2 A B^{2}\right) \leqslant A C /(2 A B)$ so $A C \geqslant 2 \cdot A B \cdot t^{*}$. Hence $B C^{2} / 4=C F^{2} \geqslant C D^{2} \geqslant 2 \cdot A B^{2} \cdot\left(t^{*}\right)^{2}+B C^{2} / 2-A B^{2} / 4$ using $(*) \Rightarrow\left(1-8\left(t^{*}\right)^{2}\right) A B^{2}$ $\geqslant B C^{2}$. Thus $t(\triangle C D F)=($ area $\triangle C D F) / C F^{2}=($ area $\triangle A B C) / B C^{2} \geqslant t^{*} /\left(1-8\left(t^{*}\right)^{2}\right)$. Now $t^{*} \geqslant 3 \hat{t} / 4$, so $t(\Delta C D F) \geqslant t^{*} /\left(1-9 \hat{t}^{2} / 2\right)>\hat{t}$, since $t^{*}>\hat{t}\left(1-9 \hat{t}^{2} / 2\right)$ and $1-$ $9 \hat{t}^{2} / 2>0$ by Lemma 1 and the definition of $\hat{t}$. Therefore $\triangle C D F$ is good. Also $\triangle D B F \sim \triangle A B C$ with $d(\triangle D B F)=d(\triangle A B C) / 2$.

We can now apply Lemma 2 to obtain $\triangle A B C$ good.

Case (v): $A D$ in $\triangle C A D, C D$ in $\triangle C D F$. As in Case (iv), $\triangle C A D$ is good. In $\triangle C D F$ bisection yields $\triangle C H F \sim \triangle B C D$ and $\triangle D F H \sim \triangle C A D$ (consequently good). In $\triangle F D B(\sim \triangle A B C)$ bisection yields $\triangle B F M \sim \triangle B C D$ and $\triangle M F D \sim \triangle C A D$ (consequently good). Applying Lemma 2 to $\triangle B C D$ now shows that $\triangle B C D$ is good. But $\triangle F D B$, obtained from $\triangle B C D$ by bisection, must then be good, so $\triangle A B C \sim \triangle F D B$ is good.

Case (vi): $C D$ in $\triangle C A D, C D$ in $\triangle C D F$. If we can show that $\triangle C A D$ is good, then repeating the argument of Case (v) shows that $\triangle A B C$ is good. We therefore analyze $\triangle C A D$.

If $C D^{2} \leqslant 3 A B^{2} / 8$, then $t(\triangle C A D)=($ area $\triangle C A D) / C D^{2} \geqslant 4 t^{*} / 3>\hat{t}$, so $\triangle C A D$ is good. Therefore assume that $C D^{2} \geqslant 3 A B^{2} / 8$. We claim that this implies that $\triangle C A D$ satisfies the hypotheses of [3, Corollary 1(i)] and is consequently good (see the remarks above).

Using $B C \leqslant A B$ in (*) gives

$$
A C^{2} / 2+A B^{2} / 2-A B^{2} / 4 \geqslant C D^{2} \geqslant 3 A B^{2} / 8 \Rightarrow A C^{2} \geqslant A B^{2} / 4, \text { i.e., } A C \geqslant A D \text {. }
$$

Thus in $\triangle C A D, C D \geqslant A C \geqslant A D$. When $\triangle C A D$ is bisected, the median formed is $A H$. To prove our claim, we must show that (a) $A D \geqslant A H$, (b) $A D \geqslant D H$, and (c) $A H \geqslant A E$ all hold.

Note that $A H^{2}=A C^{2} / 2+A D^{2} / 2-C D^{2} / 4$. Now

$$
\text { (a) } \begin{aligned}
A D^{2}-A H^{2} & =A D^{2} / 2-A C^{2} / 2+C D^{2} / 4 \\
& =A B^{2} / 16-3 A C^{2} / 8+B C^{2} / 8 \quad \text { using (*) } \\
& \geqslant 3 B C^{2} / 16-3 A C^{2} / 8 \quad \text { since } A B \geqslant B C .
\end{aligned}
$$

But $A C^{2} \leqslant C D^{2} \leqslant A C^{2} / 2+B C^{2} / 2-B C^{2} / 4$ from $(*) \Rightarrow A C^{2} \leqslant B C^{2} / 2$. Hence $A D^{2}$ $-A H^{2} \geqslant 3 B C^{2} / 16-3 B C^{2} / 16=0$ as required.

(b) $A D=A B / 2 \geqslant B C / 2 \geqslant C D / 2=D H$.

(c) $A H^{2}-A E^{2}=A C^{2} / 4+A D^{2} / 2-C D^{2} / 4$

$$
\begin{aligned}
& =A C^{2} / 8+3 A B^{2} / 16-B C^{2} / 8 \quad \text { using }(*) \\
& >0 \quad \text { since } A B \geqslant B C .
\end{aligned}
$$

This completes the proof that in all cases $\triangle A B C$ is good, and the theorem is proven. 
Remark. The above proof can also be used to derive other properties of triangles under repeated bisection. If we redefine the term "good" in such a way that

(a) all triangles generated from a good $\Delta_{01}$ are also good,

(b) Lemma 1 still holds, and

(c) Lemma 2 still holds,

then Theorem 1 is still true using exactly the same proof. In this way we now give two corollaries. It is possible to derive more quickly the inequality in the original definition of "good", using [1, Theorem 3.1] and [2, Theorem], but this method of proof does not seem to extend to proving results of the type below.

Definition. We now say that $\Delta_{01}$ is good if, identifying similar triangles, only a finite number of distinct triangles are produced from $\Delta_{01}$ by the bisection method.

It is easy to verify (a), (b) (use [2, Lemma 4]), and (c) above under this definition of "good". Consequently by the above Remark we have:

COROLLARY 1. Every triangle yields only a finite number of similarity-distinct triangles under repeated bisections.

Definition. Let $p_{k}\left(\Delta_{01}\right), k=0,1,2, \ldots$, by that fraction of the total area of $\Delta_{01}$ that after $k$ cycles of the bisection method applied to $\Delta_{01}$ is covered by triangles which satisfy the conditions of [2, Lemma 4] (these are the same as the conditions of $[3$, Corollary $1(\mathrm{i})]$ ).

On examining [2, Lemma 4], we see that $p_{k}$ is a monotonically increasing sequence. Thus $p\left(\Delta_{01}\right) \equiv \lim _{k \rightarrow \infty} p_{k}\left(\Delta_{01}\right)$ always exists.

Definition. We now say that $\Delta_{01}$ is good if $p\left(\Delta_{01}\right)=1$.

Corollary 2. For every triangle $\Delta_{01}, P\left(\Delta_{01}\right)=1$.

Proof. We must check (a), (b), and (c) of the Remark above. Clearly (a) holds as, otherwise, we have a contradiction. For (b) use [2, Lemma 4].

To prove (c) (i.e. to prove Lemma 2), we may assume that of the four triangles produced, after one cycle of the bisection method applied to $\Delta_{01}$, at least two are not similar to $\Delta_{01}$, as, otherwise, elementary calculations show that $\Delta_{01}$ satisfies the conditions of [2, Lemma 4] and so by (b) is good.

By hypothesis in Lemma 2, triangles which are not similar to $\Delta_{01}$ are good. If there are exactly two such triangles, we have

$$
p\left(\Delta_{01}\right)=1 / 4+1 / 4+p\left(\Delta^{\prime}\right) / 4+p\left(\Delta^{\prime \prime}\right) / 4
$$

where $\Delta^{\prime}, \Delta^{\prime \prime}$ are the other two triangles. But $\Delta_{01} \sim \Delta^{\prime} \sim \Delta^{\prime \prime}$, so $p\left(\Delta_{01}\right)=p\left(\Delta^{\prime}\right)=$ $p\left(\Delta^{\prime \prime}\right)$. Hence $p\left(\Delta_{01}\right)=1$. Similarly, if there are three triangles which are not similar to $\Delta_{01}$, we get $p\left(\Delta_{01}\right)=1$. This completes the proof of Lemma 2 and also the proof of Corollary 2 .

Corollary 2 is of use in explaining an apparent anomaly elsewhere [4].

Acknowledgement. The author wishes to thank the referee for his advice on making the latter part of the paper more explicit. 
Waterford Regional Technical College

Cork Road

Waterford, Ireland

1. R. B. KEARFOTT, "A proof of convergence and an error bound for the method of bisection in $R^{n}$," Math. Comp., v. 32, 1978, pp. 1147-1153.

2. I. G. ROSENBERG \& F. STENGER, "A lower bound on the angles of triangles constructed by bisecting the longest side," Math. Comp., v. 29, 1975, pp. 390-395.

3. M. STYNES, "On faster convergence of the bisection method for certain triangles," Math. Comp., v. 33, 1979, pp. 717-721.

4. M. STYNES, "Why Stenger's topological degree algorithm usually works in $R^{3}$." (In preparation.)

5. J. H. C. WHITEHEAD, “On $C^{1}$-complexes," Ann. of Math., v. 41, 1940, pp. 809-824. 